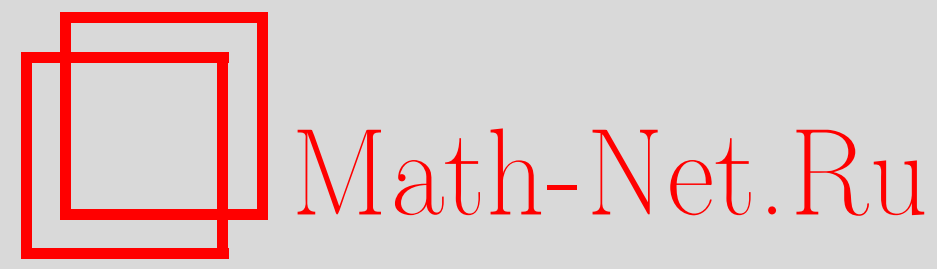

В. А. Ватутин, Е. Е. Дьяконова, Волны в редуцированных ветвящихся процессах в случайной среде, Теория вероятн. и ее примен., 2008, том 53, выпуск 4, 665-683

DOI: https://doi.org/10.4213/tvp2459

Использование Общероссийского математического портала MathNet.Ru подразумевает, что вы прочитали и согласны с пользовательским соглашением

http://www . mathnet.ru/rus/agreement

Параметры загрузки:

IP: 3.85 .183 .62

26 апреля 2023 г., 17:49:09

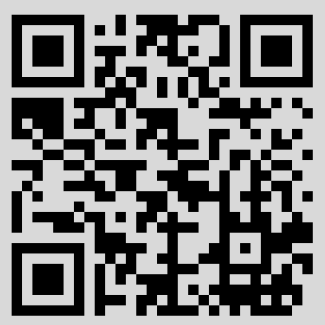




\section{ВОЛНЫ В РЕДУЦИРОВАННЫХ ВЕТВЯЩИХСЯ ПРОЦЕССАХ В СЛУЧАЙНОЙ СРЕДЕ ${ }^{1)}$}

Рассматривается ветвящийся процесс $Z(n), n=0,1, \ldots$, в случайной среде, порожденной последовательностью независимых одинаково распределенных производящих функций $f_{0}(s), f_{1}(s), \ldots$. Пусть $S_{0}=0, S_{k}=X_{1}+\cdots+X_{k}, k \geqslant 1$, - сопровождающее случайное блуждание этого процесса, где $X_{i}=\ln f_{i-1}^{\prime}(1)$, а $\tau(n)$ - самая левая точка, в которой достигается минимум блуждания $\left\{S_{k}, k \geqslant 0\right\}$ на интервале $[0, n]$. Обозначим $Z(k, m)$ число частиц, сушествовавших в ветвяшемся процессе в момент времени $k \leqslant m$ и имеющих ненулевое потомство в момент $m$. В предположении, что сопровождаюшее случайное блуждание удовлетворяет условию Дони $\mathbf{P}\left(S_{n}>0\right) \rightarrow \rho \in(0,1), n \rightarrow \infty$, доказаны (в рамках так называемого quenched approach) условные предельные теоремы при $n \rightarrow \infty$ для распределения случайной величины $Z\left(n t_{1}, n t_{2}\right)$, $0<t_{1}<t_{2}<1$, при условии $Z(n)>0$. Показано, что вид предельных распределений существенно зависит от взаимного расположения величины $\tau(n)$ и отрезка $\left[n t_{1}, n t_{2}\right]$.

Ключевые слова и фразы: ветвяшиеся процессы в случайной среде, редуцированные процессы, условие Дони, условные предельные теоремы.

1. Введение. Настоящая работа представляет собой продолжение статей [7]-[12], посвященных исследованию таких ветвящихся процессов в случайной среде (ВПСС), в которых вероятностные законы размножения частиц различных поколений независимы и одинаково распределены. Детальное построение модели рассматриваемых нами ВПСС содержится в работах [1]-[3] и [9]-[12]. Здесь мы лишь напомним основные понятия и результаты, относящиеся к этим процессам.

Пусть $\mathbf{F}=\{f(s)\}$ - множество всех производящих функций, соответствующих вероятностным распределениям с носителями, сосредоточенными на множестве неотрицательных целых чисел $\mathbf{N}_{0}$, снабженное

* Математический институт им. В. А. Стеклова РАН, ул. Губкина, 8, 119991 Москва, ГСП-1, Россия; e-mail: vatutin@mi.ras.ru, elena@mi.ras.ru

1) Работа выполнена при финансовой поддержке РФФИ (грант 05-01-00035), INTAS (проект 03-51-5018), NWO 047.016.013 и программы РАН «Современные проблемы теоретической математики». 
равномерной метрикой. Предположим, что на борелевских подмножествах пространства $\mathbf{F}$ задана вероятностная мера $\mathbb{P}$. Выбирая из множества $\mathbf{F}$ в соответствии с мерой $\mathbb{P}$ последовательность производящих функций $f_{n}(s), n=0,1, \ldots$, и фиксируя ее, мы получаем бесконечную последовательность, называемую случайной средой. Последовательность случайньх величин $\{Z(n) ; n \geqslant 0\}$, задаваемая соотношениями

$$
\begin{gathered}
Z(0)=1 \\
\mathbf{E}\left[s^{Z(n+1)} \mid f_{0}, f_{1}, \ldots, f_{n} ; Z(0), Z(1), \ldots, Z(n)\right]=\left(f_{n}(s)\right)^{Z(n)}, \quad n \geqslant 0,
\end{gathered}
$$

называется ветвяшимся процессом в случайной среде $\left\{f_{n}(s), n=\right.$ $0,1, \ldots\}$.

Пусть

$$
\begin{aligned}
f & \stackrel{d}{=} f_{0}, \quad X_{j}:=\ln f_{j-1}^{\prime}(1), \\
\eta_{j} & :=f_{j-1}^{\prime \prime}(1)\left(f_{j-1}^{\prime}(1)\right)^{-2}, \quad j \in \mathbf{N}=\{1,2, \ldots\} \\
X & \stackrel{d}{=} X_{1} ; \quad S_{0}:=0, \quad S_{n}:=X_{1}+\cdots+X_{n}, \quad n \geqslant 1 .
\end{aligned}
$$

Последовательность $\left\{S_{n} ; n \geqslant 0\right\}$ называется сопровождающим случайным блужданием, соответствуюшим ВПСС.

Далее мы предполагаем, что с вероятностью 1

$$
\limsup _{n \rightarrow \infty} S_{n}=+\infty \quad \text { и } \quad \liminf _{n \rightarrow \infty} S_{n}=-\infty \text {. }
$$

Условие (2) означает, что мы рассматриваем невырожденный критический ВПСС (см. [4] и [11]). В дальнейшем для краткости мы будем опускать слово «невырожденный» и называть процесс, удовлетворяющий (2), просто критическим ВПСС.

Обозначим $Z(k, m)$ число частиц в процессе $\{Z(n) ; n \geqslant 0\}$ в момент времени $k, 0 \leqslant k \leqslant m$, имеющих ненулевое потомство в момент времени $m$. Набор $\{Z(k, m) ; 0 \leqslant k \leqslant m<\infty\}$ называется редуцированным ветвяшимся процессом для первоначального процесса $\{Z(n) ; n \geqslant 0\}$.

Редуцированные процессы для обычных процессов ГальтонаВатсона изучали Фляйшманн и Прен [17], Зубков [14] и Фляйшманн и Зигмунд-Шультце [18]. Первые результаты для редуцированных ВПСС с дробно-линейными производящими функциями получили Боровков и Ватутин [5] и Фляйшманн и Ватутин [16], применяя так называемый annealed approach (т.е. усредняя характеристики и меры относительно распределения $\mathbb{P}$, заданного на пространстве сред). В дальнейшем $\mathrm{Ba-}$ тутин [6] обобщил результаты работы [5] и доказал, следуя annealed approach, условную предельную теорему для критических редуцированных ветвящихся процессов в случае производящих функций общего вида. 
Редуцированные ВПСС в рамках так называемого quenched approach (т.е. в ситуации, когда вероятности событий и меры рассматриваются как случайные элементы, зависящие от реализации среды) впервые исследовали Ватутин и Дьяконова в [7] и [12]. Они доказали условные предельные теоремы при $n \rightarrow \infty$ для распределения случайной величины $Z(n t, n), 0<t<1$, при условии $Z(n)>0$ в случае критического ВПСС.

В настоящей статье мы уточняем результаты упомянутых работ и доказываем условные предельные теоремы при $n \rightarrow \infty$ о распределении величины $Z\left(n t_{1}, n t_{2}\right), 0<t_{1}<t_{2}<1$, при условии $Z(n)>0$. Отложив строгие формулировки и обсуждения до следующего пункта, отметим, что результаты работ [7] и [12] указывают на то, что вид искомых предельньх распределений должен зависеть от взаимного расположения интервала $\left[n t_{1}, n t_{2}\right]$ и величины

$$
\tau(n):=\min \left\{i \in[0, n]: S_{j} \geqslant S_{i}, j=0,1, \ldots, n\right\},
$$

- самой левой точки, в которой достигается минимум случайного блуждания $\left\{S_{k} ; k \geqslant 0\right\}$ на интервале $[0, n]$.

2. Основные условия. Будем предполагать, что распределение случайной величины $X$ либо нерешетчато, либо центрально решетчато (т.е. $X$ принимает лишь значения $h k, h>0, k \in \mathbf{Z}$, и $\mathbf{P}(X=0)>0$ ) и что сопровождающее случайное блуждание удовлетворяет следующему условию Дони.

Условие $A 1$. Существует число $0<\rho<1$ такое, что

$$
\mathbf{P}\left(S_{n}>0\right) \rightarrow \rho, \quad n \rightarrow \infty .
$$

Заметим, что, согласно [13], условие $A 1$ эквивалентно формально более слабому условию Спицера

$$
\frac{1}{n} \sum_{k=1}^{n} \mathbf{P}\left(S_{k}>0\right) \longrightarrow \rho, \quad n \rightarrow \infty
$$

Пусть

$$
\gamma_{0}:=0, \quad \gamma_{j+1}:=\min \left(n>\gamma_{j}: S_{n}<S_{\gamma_{j}}\right), \quad j \geqslant 0,
$$

И

$$
\Gamma_{0}:=0, \quad \Gamma_{j+1}:=\min \left(n>\Gamma_{j}: S_{n}>S_{\Gamma_{j}}\right), \quad j \geqslant 0,
$$

- строгие убывающие и соответственно возрастающие лестничные моменты случайного блуждания $\left\{S_{n}, n \geqslant 0\right\}$. Введем функции

$$
\begin{aligned}
& V(x):=\sum_{j=0}^{\infty} \mathbf{P}\left(S_{\gamma_{j}} \geqslant-x\right), \quad x>0, \quad V(0)=1, \quad V(x)=0, \quad x<0, \\
& U(x):=1+\sum_{j=1}^{\infty} \mathbf{P}\left(S_{\Gamma_{j}}<x\right), \quad x>0, \quad U(0)=1, \quad U(x)=0, \quad x<0 .
\end{aligned}
$$


В дальнейшем мы будем отождествлять вероятностную производящую функцию

$$
f_{n}(s):=\sum_{k=0}^{\infty} \pi_{n}(k) s^{k}
$$

с бесконечномерным вектором $\pi_{n}$ :

$$
\pi_{n}:=\left\{\pi_{n}(0), \pi_{n}(1), \pi_{n}(2), \ldots\right\}, \quad \pi_{n}(k) \geqslant 0, \quad \sum_{k=0}^{\infty} \pi_{n}(k)=1, \quad n \in \mathbf{N}_{0} .
$$

Для $f(s)=\sum_{k=0}^{\infty} \pi(k) s^{k} \stackrel{d}{=} f_{n}(s)$ и $a \in \mathbf{N}_{0}$ положим

$$
\Theta(a):=\frac{\sum_{k=a}^{\infty} k^{2} \pi(k)}{\left(\sum_{k=0}^{\infty} k \pi(k)\right)^{2}}
$$

Условие $A 2$. Существуют числа $\varepsilon_{0}>0$ и $a \in \mathbf{N}_{0}$ такие, что

$$
\begin{array}{lll}
\mathbf{E}\left(\ln ^{+} \Theta(a)\right)^{1 / \rho+\varepsilon_{0}}<\infty & \text { и } & \mathbf{E}\left[V(X)\left(\ln ^{+} \Theta(a)\right)^{1+\varepsilon_{0}}\right]<\infty \\
\mathbf{E}\left(\ln ^{+} \Theta(a)\right)^{1 /(1-\rho)+\varepsilon_{0}}<\infty & \text { и } & \mathbf{E}\left[U(-X)\left(\ln ^{+} \Theta(a)\right)^{1+\varepsilon_{0}}\right]<\infty
\end{array}
$$

Более детальное обсуждение этих условий можно найти в [4] и [10]. Пусть $\Omega_{n}$ - пространство элементарных событий, соответствующее наборам $\pi_{0}, \pi_{1}, \ldots, \pi_{n-1}, Z(0), Z(1), \ldots, Z(n) ; \Omega=\Omega_{\infty}$, а

$$
\mathscr{F}_{n}=\sigma\left(\boldsymbol{\pi}_{0}, \boldsymbol{\pi}_{1}, \ldots, \boldsymbol{\pi}_{n-1} ; Z(0), Z(1), \ldots, Z(n)\right), \quad n \in \mathbf{N}_{0}, \quad \mathscr{F}:=\bar{\bigvee}_{n} \mathscr{F}_{n}
$$

- последовательность естественных $\sigma$-алгебр, порожденньх ВПСС. Мы будем использовать символы $\mathbf{E}$ и $\mathbf{P}$ для обозначения математического ожидания и вероятности относительно вероятностной меры, порождаемой исходной мерой ветвящегося процесса на набо$\operatorname{pax}\left(\pi_{0}, \pi_{1}, \ldots, \pi_{n}, \ldots ; Z(0), Z(1), \ldots, Z(n), \ldots\right)$ (за исключением некоторых случаев, которые, как мы надеемся, не приведут к недоразумениям), а символы $\mathbf{E}_{\boldsymbol{\pi}}$ и $\mathbf{P}_{\boldsymbol{\pi}}$ - для обозначения условного математического ожидания и условной вероятности при фиксированной среде $\boldsymbol{\pi}=\left(\boldsymbol{\pi}_{0}, \boldsymbol{\pi}_{1}, \ldots, \boldsymbol{\pi}_{n}, \ldots\right)$. Тройка $(\Omega, \mathscr{F}, \mathbf{P})$ является нашим основным вероятностным пространством. Нам также потребуются две копии этого пространства, обозначаемые $\left(\Omega^{-}, \mathscr{F}^{-}, \mathbf{P}^{-}\right)$и $\left(\Omega^{+}, \mathscr{F}^{+}, \mathbf{P}^{+}\right)$, на которых заданы две последовательности случайных элементов $\left\{f_{n}^{-}, n \geqslant 0\right\}$ и соответственно $\left\{f_{n}^{+}, n \geqslant 0\right\}$, порождающие соответствующие им сопровождающие случайные блуждания $\left\{S_{n}^{-}, n \geqslant 0\right\}$ и $\left\{S_{n}^{+}, n \geqslant 0\right\}$. В дальнейшем нам будет удобно все параметры и случайные величины, связанные с $\left\{f_{n}^{-}, n \geqslant 0\right\}$ и $\left\{f_{n}^{+}, n \geqslant 0\right\}$, снабжать индексами - и + соответственно. Например, мы будем использовать случайные величины

$$
\Gamma^{-}:=\min \left\{n \geqslant 1: S_{n}^{-} \geqslant 0\right\}, \quad \gamma^{+}:=\min \left\{n \geqslant 1: S_{n}^{+}<0\right\} .
$$


Положим $D=\sum_{j=1}^{\infty} j^{-1} \mathbf{P}\left(S_{j}=0\right)$ и $\mathscr{A}_{k, p}:=\left\{\Gamma^{-}>k ; \gamma^{+}>p\right\}$. Свяжем с исходными мерами $\mathbf{P}^{-}$и $\mathbf{P}^{+}$вероятностную меру $\widehat{\mathbf{P}}$ на измеримом пространстве $\left(\Omega^{-} \times \Omega^{+}, \mathscr{F}^{-} \times \mathscr{F}^{+}\right)$, полагая для $\mathscr{A}_{\in} \in \mathscr{F}_{k}^{-} \times \mathscr{F}_{p}^{+}$

$$
\widehat{\mathbf{P}}(\mathscr{A}):=e^{D} \int_{\mathscr{A}} U\left(-S_{k}^{-}\right) V\left(S_{p}^{+}\right) I\left\{\mathscr{A}_{k, p}\right\} d\left(\mathbf{P}^{-} \times \mathbf{P}^{+}\right),
$$

где $I\{\mathscr{A}\}$ - индикатор события $\mathscr{A}$ (более детальное описание этой меры, а также обоснование целесообразности ее введения можно найти в [11]). В соответствии с этим определением, мы будем использовать символ $\widehat{\mathbf{E}}$ для обозначения математического ожидания относительно меры $\widehat{\mathbf{P}}$.

3. Основные результаты. Пусть $\mathbf{M}=\mathbf{M}([0, \infty))$ - множество всех (возможно, несобственных) вероятностных мер на $[0, \infty)$, снабженное топологией слабой сходимости. Для меры $\mathscr{M} \in \mathbf{M}$ и функции $J: \mathbf{R} \rightarrow \mathbf{R}$ положим $\mathscr{M}[J]:=\int J(x) \mathscr{M}(d x)$, если указанный интеграл сушествует. В дальнейшем мы будем рассматривать вероятностные распределения на $\mathbf{M}$, индуцированные значениями вероятностньх мер $\mathbf{P}$ и соответственно $\widehat{\mathbf{P}}$, на реализациях случайной среды. Способ построения таких распределений будет ясен из последующего изложения. Такой подход позволит нам трактовать элементы множества $\mathbf{M}$ как случайные меры и даст возможность изучать условные распределения случайных мер на $\mathbf{M}$ при условии осуществления интересующих нас событий $A$ из естественной $\sigma$-алгебры на $\mathbf{M}$. Для мер $\mathscr{M}^{(n)}$ и $\mathscr{M}$ из $\mathbf{M}$ мы будем использовать выражение

$$
\mathscr{M}^{(n)} \stackrel{w_{A}(\mathbf{P}, \widehat{\mathbf{P}})}{\longrightarrow} \mathscr{M}, \quad n \rightarrow \infty,
$$

для обозначения следуюшего вида условной слабой сходимости случайных мер $\mathscr{M}^{(n)}$ к случайной мере $\mathscr{M}$ при $n \rightarrow \infty$ : для любых неслучайных непрерывных функций $J, g: \mathbf{R} \rightarrow \mathbf{R}$ с компактными носителями

$$
\mathbf{E}\left[g\left(\mathscr{M}^{(n)}[J]\right) \mid \mathscr{M}^{(n)} \in A\right] \longrightarrow \widehat{\mathbf{E}}[g(\mathscr{M}[J])], \quad n \rightarrow \infty .
$$

При $A=\mathbf{M}$ условная сходимость преврашается в безусловную, и в этом случае вместо (4) мы будем писать

$$
\mathscr{M}^{(n)} \stackrel{w(\mathbf{P}, \widehat{\mathbf{P}})}{\longmapsto} \mathscr{M}, \quad n \rightarrow \infty .
$$

Сопоставим мерам $\mathscr{M}^{(n)} \in \mathbf{M}, n \in \mathbf{N}_{0}$, и $\mathscr{M} \in \mathbf{M}$ их преобразования Лапласа

$$
M^{(n)}(\lambda):=\int_{0}^{\infty} e^{-\lambda x} \mathscr{M}^{(n)}(d x), \quad M(\lambda):=\int_{0}^{\infty} e^{-\lambda x} \mathscr{M}(d x), \quad \lambda \in[0, \infty) .
$$

С мерами $\mathscr{M}^{(n)}$ и $\mathscr{M}$ из $\mathbf{M}$, носители которых сконцентрированы на множестве $\mathbf{N}_{0}$, мы свяжем их производящие функции

$$
m^{(n)}(s):=\sum_{k=0}^{\infty} \mathscr{M}^{(n)}(k) s^{k}, \quad m(s):=\sum_{k=0}^{\infty} \mathscr{M}(k) s^{k}
$$


(строго говоря, здесь нужно было бы писать $\mathscr{M}^{(n)}(\{k\})$ и $\mathscr{M}(\{k\})$, но мы не будем использовать такие обозначения, чтобы избежать излишне громоздких формул).

Чтобы отличать меры, носители которых целиком лежат в $\mathbf{N}_{0}$, от мер, не обладающих таким свойством, нам будет иногда удобно вместо (4) использовать эквивалентные записи

$$
m^{(n)}(s) \stackrel{w_{A}\left(\mathbf{P}_{\vec{P}} \widehat{\mathbf{P}}\right)}{\longmapsto} m(s), \quad n \rightarrow \infty,
$$

если носители мер $\mathscr{M}^{(n)}$ и $\mathscr{M}$ целиком сосредоточены в $\mathrm{N}_{0}$, и

$$
M^{(n)}(\lambda) \stackrel{w_{A}(\mathbf{P} \widehat{\mathbf{P}})}{\longmapsto} M(\lambda), \quad n \rightarrow \infty,
$$

если носители мер $\mathscr{M}^{(n)}$ и $\mathscr{M}$ не сконцентрированы полностью в $\mathbf{N}_{0}$.

Эти же обозначения будут использоваться и в других сходных ситуациях.

Для $0 \leqslant k \leqslant n$ положим

$$
\beta_{n}(k):=\frac{1}{\mathbf{E}_{\pi}[Z(k, n) \mid Z(n)>0]}=\frac{1-f_{0, n}(0)}{e^{S_{k}}\left(1-f_{k, n}(0)\right)} .
$$

В частности,

$$
\mathbf{E}_{\pi}[Z(n) \mid Z(n)>0]=\frac{e^{S_{n}}}{1-f_{0, n}(0)}=\frac{1}{\beta_{n}(n)} .
$$

Обозначим

$$
\begin{aligned}
L^{(n)}(\lambda) & :=\mathbf{E}_{\pi}\left[e^{-\lambda Z(n) \beta_{n}(n)} \mid Z(n)>0\right] \\
& =\int_{0}^{\infty} e^{-\lambda x} \mathbf{P}_{\pi}\left(Z(n) \beta_{n}(n) \in d x \mid Z(n)>0\right)=: \int_{0}^{\infty} e^{-\lambda x} \mathscr{L}^{(n)}(d x) .
\end{aligned}
$$

Пусть $\mathscr{W}^{(n)}(d x)=x \mathscr{L}^{(n)}(d x)$ и

$$
W^{(n)}(\lambda):=\int_{0}^{\infty} e^{-\lambda x \mathscr{W}^{(n)}}(d x)=-\frac{d}{d \lambda} L^{(n)}(\lambda) .
$$

Следующее утверждение является комбинацией нескольких результатов, доказанных в [10] (см. следствие 1, теорему 1 и лемму 7 упомянутой работы).

Теорема 1. Если выполнень условия $A 1$ и $A 2$, то существует случайная мера $\mathscr{L}$ (являющаяся с вероятностью 1 собственной $u$ невырожденной) такая, что

$$
L^{(n)}(\lambda) \stackrel{w(\mathbf{P}, \widehat{\mathbf{P}})}{\longmapsto} L(\lambda):=\int_{0}^{\infty} e^{-\lambda x} \mathscr{L}(d x), \quad n \rightarrow \infty .
$$

Более того, если $\mathscr{W}(x)=x \mathscr{L}(d x)$, mo $п р и ~ n \rightarrow \infty$

$$
W^{(n)}(\lambda) \stackrel{w(\mathbf{P}, \widehat{\mathbf{P}})}{\longrightarrow} W(\lambda):=\int_{0}^{\infty} e^{-\lambda x} \mathscr{W}(d x)=-\frac{d L(\lambda)}{d \lambda} .
$$


Выберем два числа $0<t_{1}<t_{2}<1$ и начиная с этого момента будем считать, что они фиксированы раз и навсегда. Кроме того, условимся подразумевать под произведением вида $n t, t \in(0,1)$, целое число $[n t]$. Положим

$$
\begin{aligned}
f_{k, n}(s):=f_{k}\left(f_{k+1}\left(\cdots\left(f_{n-1}(s)\right) \cdots\right)\right), & & 0 \leqslant k \leqslant n-1, \quad f_{n, n}(s):=s, \\
f_{n, m}(s):=f_{n-1}\left(f_{n-2}\left(\cdots\left(f_{m}(s)\right) \cdots\right)\right), & & n \geqslant m+1 .
\end{aligned}
$$

Для выбранных выше чисел $0<t_{1}<t_{2}<1$ и величины

введем меру

$$
\Theta_{n}\left(t_{1}, t_{2}\right):=\frac{1-f_{0, n t_{1}}(0)}{e^{S_{n t_{1}}}\left(1-f_{n t_{1}, n t_{2}}(0)\right)}
$$

$$
\mathscr{M}^{(n)}(d x)=\mathscr{M}_{t_{1}, t_{2}}^{(n)}(d x):=\mathbf{P}_{\pi}\left[Z\left(n t_{1}, n t_{2}\right) \Theta_{n}\left(t_{1}, t_{2}\right) \in d x \mid Z(n)>0\right]
$$

с преобразованием Лапласа

$$
M_{t_{1}, t_{2}}^{(n)}(\lambda):=\mathbf{E}_{\pi}\left[s^{-\lambda Z\left(n t_{1}, n t_{2}\right) \Theta_{n}\left(t_{1}, t_{2}\right)} \mid Z(n)>0\right]=\int_{0}^{\infty} e^{-\lambda x} \mathscr{M}_{t_{1}, t_{2}}^{(n)}(d x) .
$$

Зададим также меру

$$
\mu^{(n)}(k):=\mu_{t_{1}, t_{2}}^{(n)}(k)=\mathbf{P}_{\pi}\left[Z\left(n t_{1}, n t_{2}\right)=k \mid Z(n)>0\right], \quad k \in \mathbf{N}_{0},
$$

c производящей функцией

$$
m^{(n)}(s)=m_{t_{1}, t_{2}}^{(n)}(s):=\mathbf{E}_{\pi}\left[s^{Z\left(n t_{1}, n t_{2}\right)} \mid Z(n)>0\right]:=\sum_{k=1}^{\infty} \mu^{(n)}(k) s^{k} .
$$

В последуюших рассуждениях существенное значение имеют следующие четыре события:

$$
\begin{aligned}
& A_{1}=A_{1}(n):=\left\{\tau(n)<n t_{1}\right\} \\
& A_{2}=A_{2}(n):=\left\{\tau(n) \in\left[n t_{1}, n t_{2}\right]\right\} \\
& A_{3}=A_{3}(n):=\left\{\tau(n)>n t_{2}, \tau\left(n t_{2}\right) \leqslant n t_{1}\right\} \\
& A_{4}=A_{4}(n):=\left\{\tau(n)>n t_{2}, \tau\left(n t_{2}\right)>n t_{1}\right\} .
\end{aligned}
$$

Теперь мы можем сформулировать условные предельные теоремы (в рамках quenched approach) о распределении процесса $Z\left(n t_{1}, n t_{2}\right)$ при условии осуществления одного из событий $A_{i}(n), i=1,2,3,4$.

Теорема 2. Если выполнены условия $A 1$ и $A 2$, то

$$
M_{t_{1}, t_{2}}^{(n)}(\lambda) \stackrel{w_{A_{1}}(\mathbf{P}, \widehat{\mathbf{P}})}{\longmapsto} L(\lambda) \quad n p u \quad n \rightarrow \infty,
$$

где $L(\lambda)$ - преобразование Лапласа, упомянутое в теореме 1.

Теорема 3. При выполнении условий $A 1$ и $A 2$,

$$
M_{t_{1}, t_{2}}^{(n)}(\lambda) \stackrel{w_{A_{3}}(\mathbf{P}, \widehat{\mathbf{P}})}{\longrightarrow} W(\lambda) \quad n p u \quad n \rightarrow \infty,
$$

где $W(\lambda)$ - преобразование Лапласа, упомянутое в теореме 1 . 
Теорема 4. Если выполнены условия $A 1$ и $A 2$, то для $i=2$ и $i=4$

$$
m_{t_{1}, t_{2}}^{(n)}(s) \stackrel{w_{A_{i}}(\mathbf{P}, \widehat{\mathbf{P}})}{\longmapsto} s \quad n p u \quad n \rightarrow \infty
$$

Для того, чтобы дать неформальную интерпретацию наших результатов, напомним, что (см. $[15$, гл. IV,$\S 20])$ при выполнении условия $A 1$

$$
-n^{-1} \tau(n) \stackrel{d}{\longmapsto} \tau, \quad n \rightarrow \infty
$$

где $\tau$ - случайная величина, удовлетворяющая обобшенному закону арксинуса с параметром $\rho$ :

$$
\mathbf{P}(\tau \leqslant x)=\frac{\sin \pi \rho}{\pi} \int_{0}^{x} \frac{d y}{y^{1-\rho}(1-y)^{\rho}}, \quad x \in[0,1] .
$$

Кроме того, в соответствии с теоремой 1 работы [10], существует положительная и конечная с вероятностью 1 случайная величина $\widehat{\Theta}$ такая, что

$$
\Theta_{n}\left(t_{1}, t_{2}\right) \exp \left\{S_{\tau\left(n t_{1}, n t_{2}\right)}-S_{\tau\left(n t_{1}\right)}\right\} \stackrel{d}{\longmapsto} \widehat{\Theta}, \quad n \rightarrow \infty,
$$

для любых $0<t_{1}<t_{2}<1$, где

$$
\tau\left(n t_{2}, n t_{1}\right)=\min \left\{i \in\left(n t_{1}, n t_{2}\right]: S_{j} \geqslant S_{i}, j=n t_{1}+1, \ldots, n t_{2}\right\} .
$$

Выберем теперь достаточно «большое» $n$, зафиксируем реализацию среды $\left(\boldsymbol{\pi}_{0}, \boldsymbol{\pi}_{1}, \ldots, \boldsymbol{\pi}_{n}\right)$ и рассмотрим случайную величину $Z\left(n t_{1}, n t_{2}\right)$, равную числу частиц, существовавших в момент времени $n t_{1}$ и имеющих ненулевое потомство в момент времени $n t_{2}$. Перемещая точки $t_{1}, t_{2}$ по интервалу $[0,1]$ справа налево, с соблюдением условия $t_{1} \leqslant t_{2}$, и начиная движение с точек $t_{1}=t_{2}=1$, мы будем наблюдать следующую типичную картину.

Если $\tau(n)<n t_{1}$, то $\tau(n)=\tau\left(n t_{1}\right)$, и следовательно, согласно соотношению (8) и теореме $2, Z\left(n t_{1}, n t_{2}\right) \asymp \exp \left\{S_{\tau\left(n t_{1}, n t_{2}\right)}-S_{\tau(n)}\right\}$ при $n \rightarrow \infty$.

Если $\tau(n) \in\left[n t_{1}, n t_{2}\right]$, то $\mathbf{P}_{\pi}\left(Z\left(n t_{1}, n t_{2}\right)=1\right) \approx 1$ при $n \rightarrow \infty$. Это, нестрого говоря, означает, что все частицы, существуюшие в процессе $\{Z(k), k \geqslant 0\}$ в момент времени $n t_{2}$, являются потомками одной частицы, сушествовавшей в момент времени $n t_{1}$. Аналогичная картина наблюдается и при $\left\{\tau(n)>n t_{2}>\tau\left(n t_{2}\right)>n t_{1}\right\}$.

И наконец, если $\left\{\tau(n)>n t_{2}>n t_{1} \geqslant \tau\left(n t_{2}\right)\right\}$, то $\tau\left(n t_{2}\right)=\tau\left(n t_{1}\right)$, что в силу (8) и теоремы 3 приводит к соотношению $Z\left(n t_{1}, n t_{2}\right) \asymp$ $\exp \left\{S_{\tau\left(n t_{1}, n t_{2}\right)}-S_{\tau\left(n t_{1}\right)}\right\}$ при $n \rightarrow \infty$.

Таким образом, при смещении точек $t_{1} \leqslant t_{2}$ от 1 к 0 , величина $Z\left(n t_{1}, n t_{2}\right)$ меняется волнообразно. При этом логарифмы амплитуд волн определяются (с точностью до случайных констант) разностями $S_{\tau\left(n t_{1}, n t_{2}\right)}-S_{\tau\left(n t_{1}\right)}$. 
4. Вспомогательные утверждения. Для $k \leqslant n$ обозначим

$$
\sigma_{n}(k):=\frac{e^{S_{k}}\left(1-f_{k, n}(0)\right)}{1-f_{0, n}(0)} .
$$

Лемма 1. Если выполненъ условия $A 1$ и $A 2$, то для фиксированного $0<t<1$

$$
\left\{\sigma_{n}(n t) \mid \tau(n)>n t\right\} \stackrel{d}{\longrightarrow} 1 .
$$

Для доказательства этого утверждения достаточно применить следствие 4 работы [10] с $\beta_{n}(k)=1 / \sigma_{n}(k)$.

$\mathrm{B}$ дальнейшем для краткости мы будем использовать следующие обозначения:

$$
\begin{gathered}
a:=f_{n t_{1}, n t_{2}}(0) \\
y_{1}:=1-a=1-f_{n t_{1}, n t_{2}}(0), \quad y_{2}:=f_{n t_{1}, n}(0)-f_{n t_{1}, n t_{2}}(0) .
\end{gathered}
$$

Лемма 2. Справедливо представление

$$
m^{(n)}(s)=m_{t_{1}, t_{2}}^{(n)}(s)=\frac{f_{0, n t_{1}}\left(a+s y_{1}\right)-f_{0, n t_{1}}\left(a+s y_{2}\right)}{1-f_{0, n}(0)} .
$$

Доказательст в о. Ясно, что

$$
m^{(n)}(s)=\frac{\mathbf{E}_{\pi}\left[s^{Z\left(n t_{1}, n t_{2}\right)}\right]-\mathbf{E}_{\pi}\left[s^{Z\left(n t_{1}, n t_{2}\right)} I\{Z(n)=0\}\right]}{1-f_{0, n}(0)}, \quad s \in[0,1) .
$$

Несложно также видеть, что

$$
\mathbf{E}_{\pi}\left[s^{Z\left(n t_{1}, n t_{2}\right)}\right]=f_{0, n t_{1}}\left(f_{n t_{1}, n t_{2}}(0)+s\left(1-f_{n t_{1}, n t_{2}}(0)\right)\right) .
$$

С другой стороны, для $s_{1} \in[0,1], s_{2} \in[0,1]$

$$
\mathbf{E}_{\pi}\left[s_{1}^{Z\left(n t_{1}, n t_{2}\right)} s_{2}^{Z\left(n t_{2}\right)} I\{Z(n)=0\}\right]=\mathbf{E}_{\pi}\left[s_{1}^{Z\left(n t_{1}, n t_{2}\right)}\left(s_{2} f_{n t_{2}, n}(0)\right)^{Z\left(n t_{2}\right)}\right]
$$

и

$$
\begin{aligned}
& \mathbf{E}_{\pi}\left[s_{1}^{Z\left(n t_{1}, n t_{2}\right)} s_{2}^{Z\left(n t_{2}\right)}\right] \\
& \quad=f_{0, n t_{1}}\left(f_{n t_{1}, n t_{2}}(0)+\left(1-f_{n t_{1}, n t_{2}}(0)\right) s_{1} \frac{f_{n t_{1}, n t_{2}}\left(s_{2}\right)-f_{n t_{1}, n t_{2}}(0)}{1-f_{n t_{1}, n t_{2}}(0)}\right) .
\end{aligned}
$$

Отсюда, полагая $s_{1}=s$ и $s_{2}=1$ в (15), получаем

$$
\mathbf{E}_{\pi}\left[s^{Z\left(n t_{1}, n t_{2}\right)} I\{Z(n)=0\}\right]=f_{0, n t_{1}}\left(f_{n t_{1}, n t_{2}}(0)+s\left(f_{n t_{1}, n}(0)-f_{n t_{1}, n t_{2}}(0)\right)\right) .
$$

Соотношения (13)-(16) дают (12).

Лемма доказана.

Введем функцию

$$
m^{(n) *}(s)=m_{t_{1}, t_{2}}^{(n) *}(s):=s \frac{f_{0, n t_{1}}^{\prime}\left(a+s y_{1}\right)}{e^{S_{n t_{1}}}}=: \sum_{k=1}^{\infty} \mu^{(n) *}(k) s^{k}
$$


и обозначим

$$
d\left(\mu^{(n)}, \mu^{(n) *}\right):=\frac{1}{2} \sum_{k=1}^{\infty}\left|\mu^{(n)}(k)-\mu^{(n) *}(k)\right|
$$

расстояние по вариации между мерами $\mu^{(n)}$ и $\mu^{(n) *}$.

Лемма 3. Если выполнены условия $A 1 u A 2$, то для любого $\varepsilon>0$

$$
\lim _{n \rightarrow \infty} \mathbf{P}\left(d\left(\mu^{(n)}, \mu^{(n) *}\right)>\varepsilon \mid \tau(n)>n t_{1}\right)=0 .
$$

Д оказате льст в о. Запишем $f_{0, n t_{1}}(s)$ в виде

$$
f_{0, n t_{1}}(s)=\sum_{h=0}^{\infty} \mathscr{F}^{(n)}(h) s^{h} .
$$

Из леммы 2 и определений (17) и (18) следует, что

$$
\begin{aligned}
m^{(n)}(s)\left(1-f_{0, n}(0)\right) & =f_{0, n t_{1}}\left(a+y_{1} s\right)-f_{0, n t_{1}}\left(a+y_{2} s\right) \\
& =\sum_{h=1}^{\infty} \mathscr{F}^{(n)}(h) \sum_{k=1}^{h} C_{h}^{k} s^{k} a^{h-k}\left(y_{1}^{k}-y_{2}^{k}\right) \\
& =\sum_{k=1}^{\infty} s^{k}\left(y_{1}^{k}-y_{2}^{k}\right) \sum_{h=k}^{\infty} \mathscr{F}^{(n)}(h) C_{h}^{k} a^{h-k}
\end{aligned}
$$

и

$$
\begin{aligned}
m^{(n) *}(s) e^{S_{n t_{1}}} & =\sum_{h=1}^{\infty} h \mathscr{F}^{(n)}(h) s\left(a+y_{1} s\right)^{h-1} \\
& =\sum_{h=1}^{\infty} h \mathscr{F}^{(n)}(h) \sum_{k=1}^{h} C_{h-1}^{k-1} s^{k} y_{1}^{k-1} a^{h-k} \\
& =\sum_{k=1}^{\infty} s^{k} y_{1}^{k-1} \sum_{h=k}^{\infty} h \mathscr{F}^{(n)}(h) C_{h-1}^{k-1} a^{h-k} \\
& =\sum_{k=1}^{\infty} s^{k} y_{1}^{k-1} k \sum_{h=k}^{\infty} \mathscr{F}^{(n)}(h) C_{h}^{k} a^{h-k}
\end{aligned}
$$

Объединяя (5), (17), (19) и (20), находим

$$
\begin{aligned}
\mu^{(n)}(k) & =\frac{y_{1}^{k}-y_{2}^{k}}{1-f_{0, n}(0)} \sum_{h=k}^{\infty} \mathscr{F}^{(n)}(h) C_{h}^{k} a^{h-k} \\
\mu^{(n) *}(k) & =k y_{1}^{k-1} e^{-S_{n t_{1}}} \sum_{h=k}^{\infty} \mathscr{F}^{(n)}(h) C_{h}^{k} a^{h-k} .
\end{aligned}
$$

Поскольку $0 \leqslant y_{2} \leqslant y_{1} \leqslant 1$, то

$$
k y_{1}^{k-1}\left(y_{1}-y_{2}\right)-\frac{k(k-1)}{2} y_{1}^{k-2}\left(y_{1}-y_{2}\right)^{2} \leqslant y_{1}^{k}-y_{2}^{k} \leqslant k y_{1}^{k-1}\left(y_{1}-y_{2}\right)
$$


Используя эти оценки, равенство $y_{1}-y_{2}=1-f_{n t_{1}, n}(0)$ и определение (9), получаем

$$
\frac{y_{1}^{k}-y_{2}^{k}}{1-f_{0, n}(0)}-k y_{1}^{k-1} e^{-S_{n t_{1}}} \leqslant k y_{1}^{k-1} e^{-S_{n t_{1}}}\left(\sigma_{n}\left(n t_{1}\right)-1\right)
$$

и

$$
\begin{aligned}
& k y_{1}^{k-1} e^{-S_{n t_{1}}}\left(\sigma_{n}\left(n t_{1}\right)-1\right)-\frac{k(k-1)}{2} y_{1}^{k-2} \frac{\left(1-f_{n t_{1}, n}(0)\right)^{2}}{1-f_{0, n}(0)} \\
& \quad \leqslant \frac{y_{1}^{k}-y_{2}^{k}}{1-f_{0, n}(0)}-k y_{1}^{k-1} e^{-S_{n t_{1}}} .
\end{aligned}
$$

Отсюда следует, что

$$
\begin{gathered}
\left|\mu^{(n)}(k)-\mu^{(n) *}(k)\right| \leqslant e^{-S_{n t_{1}}}\left(\sigma_{n}\left(n t_{1}\right)-1\right) \sum_{h=k}^{\infty} \mathscr{F}^{(n)}(h) C_{h}^{k} a^{h-k} y_{1}^{k-1} k \\
+\frac{\left(1-f_{n t_{1}, n}(0)\right)^{2}}{1-f_{0, n}(0)} \sum_{h=k}^{\infty} \mathscr{F}^{(n)}(h) C_{h}^{k} a^{h-k} y_{1}^{k-2} \frac{k(k-1)}{2}
\end{gathered}
$$

Заметим теперь, что в силу равенства $a+y_{1}=1$

$$
\begin{aligned}
& \sum_{k=1}^{\infty} \sum_{h=k}^{\infty} \mathscr{F}^{(n)}(h) C_{h}^{k} a^{h-k} y_{1}^{k-1} k=\sum_{h=1}^{\infty} \mathscr{F}^{(n)}(h) \sum_{k=1}^{h} C_{h}^{k} a^{h-k} y_{1}^{k-1} k \\
& =\sum_{h=1}^{\infty} \mathscr{F}^{(n)}(h) h\left(a+y_{1}\right)^{h-1}=\sum_{h=1}^{\infty} \mathscr{F}^{(n)}(h) h=\mathbf{E}_{\pi}\left[Z\left(n t_{1}\right)\right]=e^{S_{n t_{1}}}
\end{aligned}
$$

и, аналогично,

$$
\sum_{k=1}^{\infty} \sum_{h=k}^{\infty} \mathscr{F}^{(n)}(h) C_{h}^{k} a^{h-k} y_{1}^{k-2} \frac{k(k-1)}{2}=\frac{1}{2} \mathbf{E}_{\pi}\left[Z\left(n t_{1}\right) Z\left(n t_{1}-1\right)\right] .
$$

Нетрудно проверить (см. $[11$, с. 39$])$, что

$$
\mathbf{E}_{\pi}\left[Z\left(n t_{1}\right) Z\left(n t_{1}-1\right)\right]=\left(f_{0, n t_{1}}(s)\right)_{s=1}^{\prime \prime}=2 e^{2 S_{n t_{1}}} b_{n t_{1}}
$$

где (см. (1))

$$
b_{r}:=\frac{1}{2} \sum_{k=0}^{r-1} \frac{f_{k}^{\prime \prime}(1)}{\left(f_{k}^{\prime}(1)\right)^{2}} e^{-S_{k}}=\frac{1}{2} \sum_{k=0}^{r-1} \eta_{k+1} e^{-S_{k}}
$$

Так как

$$
\begin{aligned}
\frac{\left(1-f_{n t_{1}, n}(0)\right)^{2}}{2\left(1-f_{0, n}(0)\right)} \mathbf{E}_{\pi}\left[Z\left(n t_{1}\right) Z\left(n t_{1}-1\right)\right] & =\sigma_{n}^{2}\left(n t_{1}\right)\left(1-f_{0, n}(0)\right) b_{n t_{1}} \\
& \leqslant \sigma_{n}^{2}\left(n t_{1}\right) e^{S_{\tau(n)}} b_{n t_{1}}
\end{aligned}
$$


то, суммируя (21) по $k$ от 1 до $\infty$ и используя предыдущие оценки, нетрудно установить, что

$$
2 d\left(\mu^{(n)}, \mu^{(n) *}\right) \leqslant \sigma_{n}\left(n t_{1}\right)-1+\sigma_{n}^{2}\left(n t_{1}\right) e^{S_{\tau(n)}} b_{n t_{1}} .
$$

Заметим наконец, что для любого целого $N$ и любого $\varepsilon>0$

$$
\begin{aligned}
& \mathbf{P}\left(e^{S_{\tau(n)}} b_{n t_{1}}>\varepsilon ; \tau(n)>n t_{1}\right) \\
& \quad \leqslant \mathbf{P}\left(n t_{1}+N>\tau(n)>n t_{1}\right)+\mathbf{P}\left(e^{S_{\tau(n)}} b_{\tau(n)-N}>\varepsilon\right)
\end{aligned}
$$

где первый член в правой части (26) пренебрежимо мал при $n \rightarrow \infty$ согласно обобшенному закону арксинуса, в то время как

$$
\lim _{N \rightarrow \infty} \limsup _{n \rightarrow \infty} \mathbf{P}\left(e^{S_{\tau(n)}} b_{\tau(n)-N}>\varepsilon\right)=0
$$

по лемме 15 из [10]. Следовательно, для любого $\varepsilon>0$

$$
\lim _{n \rightarrow \infty} \mathbf{P}\left(e^{S_{\tau(n)}} b_{n t_{1}}>\varepsilon ; \tau(n)>n t_{1}\right)=0 .
$$

Отсюда, используя лемму 1 , получаем, что при выполнении условия $\tau(n)>n t_{1}$ величина $\sigma_{n}^{2}\left(n t_{1}\right) e^{S_{\tau(n)}} b_{n t_{1}}$ стремится к нулю по вероятности при $n \rightarrow \infty$. Вспоминая теперь оценку (24) и применяя лемму 1 , заключаем, что при выполнении условия $\tau(n)>n t_{1}$ правая часть (25) стремится к нулю по вероятности при $n \rightarrow \infty$.

Лемма доказана.

Рассмотрим теперь пару последовательностей случайной среды $\left\{f_{n}^{-}, f_{n}^{+} ; n \geqslant 0\right\}$, заданную на измеримом пространстве $\left(\Omega^{-} \times \Omega^{+}\right.$, $\left.\mathscr{F}^{-} \times \mathscr{F}^{+}\right)$, и соответствуюшую ей пару сопровождающих случайных блужданий $\left\{S_{n}^{-}, S_{n}^{+} ; n \geqslant 0\right\}$. Следующее утверждение, связываюшее свойства этих двух сред, было доказано в [10]:

Если выполнень условия $A 1$ и $A 2$ и

$$
\zeta_{l, m}^{-}(s):=\frac{1-f_{l, m}^{-}(s)}{e^{S_{l}^{-}-S_{m}^{-}}}, \quad m \in \mathbf{N}_{0}, \quad l \geqslant m+1,
$$

то $\widehat{\mathbf{P}}$-п.н. существует и является положительным предел

$$
\zeta:=\lim _{\min (l, r) \rightarrow \infty} \zeta_{l, 0}^{-}\left(f_{0, r}^{+}(0)\right) \leqslant 1 .
$$

Для $l, m, k \in \mathbf{N}$ c $m \leqslant k$ введем обозначения

$$
\begin{aligned}
\Gamma(l, m, k) & :=\frac{e^{-S_{m}^{+}-S_{l}^{-}}\left(1-f_{l, 0}^{-}\left(f_{0, k}^{+}(0)\right)\right)}{1-f_{m, k}^{+}(0)}=\frac{e^{-S_{m}^{+}} \zeta_{l, 0}^{-}\left(f_{0, k}^{+}(0)\right)}{1-f_{m, k}^{+}(0)} \\
\Theta(l, m, k) & :=\frac{e^{-S_{m}^{+}-S_{l}^{-}}\left(1-f_{l, 0}^{-}\left(f_{0, m}^{+}(0)\right)\right)}{1-f_{m, k}^{+}(0)}=\frac{e^{-S_{m}^{+} \zeta_{l, 0}^{-}\left(f_{0, m}^{+}(0)\right)}}{1-f_{m, k}^{+}(0)} .
\end{aligned}
$$


Лемма 4. Если выполнены условия $A 1$ и $A 2$, то

$$
\lim _{m \rightarrow \infty} \Theta(l, m, k)=0 \quad \widehat{\mathbf{P}}-\text { n.н. }
$$

Д оказатель ст в о. Несложно видеть, что

$$
\Theta(l, m, k) \leqslant e^{-S_{m}^{+}}\left(1-f_{m, k}^{+}(0)\right)^{-1}
$$

Применяя рассуждения, близкие к тем, которые использовались при доказательстве леммы 2 в [12], можно показать, что правая часть неравенства (29) стремится к нулю $\widehat{\mathbf{P}}$-п.н. при $m \rightarrow \infty$.

Лемма доказана.

Для $l, m, k \in \mathbf{N}$, где $m \leqslant k$, положим

$$
\begin{aligned}
& R_{m}^{(l, k)}(\lambda):=1-\frac{1-f_{l, 0}^{-}\left(f_{0, m}^{+}\left(1-\left(1-e^{-\lambda \Gamma(l, m, k)}\right)\left(1-f_{m, k}^{+}(0)\right)\right)\right)}{1-f_{l, 0}^{-}\left(f_{0, k}^{+}(0)\right)}, \\
& T_{m}^{(l, k)}(\lambda):=1-\frac{1-f_{l, 0}^{-}\left(f_{0, m}^{+}\left(1-\left(1-e^{-\lambda \Theta(l, m, k)}\right)\left(1-f_{m, k}^{+}(0)\right)\right)\right)}{1-f_{l, 0}^{-}\left(f_{0, m}^{+}(0)\right)} .
\end{aligned}
$$

Несложно видеть, что $T_{m}^{(l, k)}(\lambda)$ является преобразованием Лапласа некоторой вероятностной меры $\mathscr{T}_{m}^{(l, k)}$ :

$$
T_{m}^{(l, k)}(\lambda)=\int_{0}^{\infty} e^{-\lambda x} \mathscr{T}_{m}^{(l, k)}(d x) .
$$

Лемма 5. Если условия $A 1$ и $A 2$ выполнены, то для любого $\lambda>0$

$$
\lim _{\min (l, m) \rightarrow \infty} T_{m}^{(l, k)}(\lambda)=L(\lambda) \quad \widehat{\mathbf{P}}-\text { n.н. }
$$

Д ок а з а т е л с т в о. В силу леммы 3 работы [12]

$$
\lim _{\min (l, m) \rightarrow \infty} R_{m}^{(l, k)}(\lambda)=L(\lambda) \quad \widehat{\mathbf{P}} \text {-п.н. }
$$

Так как $m \leqslant k$, то из $(28)$ следует, что $\widehat{\mathbf{P}}$-п.н.

$$
\lim _{\min (l, m) \rightarrow \infty} \frac{\Theta(l, m, k)}{\Gamma(l, m, k)}=\lim _{\min (l, m) \rightarrow \infty} \frac{\zeta_{l, 0}^{-}\left(f_{0, m}^{+}(0)\right)}{\zeta_{l, 0}^{-}\left(f_{0, k}^{+}(0)\right)}=1 .
$$

Применяя рассуждения, сходные с теми, которые использовались при доказательстве леммы 3 в [12], можно показать при помощи (32) и леммы 4, что для любого $\lambda>0 \widehat{\mathbf{P}}$-п.н.

$$
\lim _{\min (l, m) \rightarrow \infty} \frac{T_{m}^{(l, k)}(\lambda)}{R_{m}^{(l, k)}(\lambda)}=1 .
$$

Теперь утверждение леммы вытекает из соотношений (31)-(33).

Обозначим

$$
T_{m}^{(l, k) *}(\lambda):=-\frac{d}{d \lambda} T_{m}^{(l, k)}(\lambda)
$$


Лемма 6. Если выполнены условия $A 1$ и $A 2$, то для любого $\lambda>0$

$$
\lim _{\min (l, m) \rightarrow \infty} T_{m}^{(l, k) *}(\lambda) e^{-\lambda \Theta(l, m, k)}=-L^{\prime}(\lambda) \quad \widehat{\mathbf{P}}_{-} \text {-n.н. }
$$

Д о к азате ль с т в о. Заметим, что $T_{m}^{(l, k)}(\lambda)$ и $L(\lambda)$ являются аналитическими функциями в области $\operatorname{Re} \lambda>0$. Опираясь на этот факт и используя лемму 5 и соотношение (34), получаем, что для любого $\lambda>0$

$$
\lim _{\min (l, m) \rightarrow \infty} T_{m}^{(l, k) *}(\lambda)=-L^{\prime}(\lambda) \quad \widehat{\mathbf{P}} \text {-п.н. }
$$

Утверждение леммы вытекает теперь из (35) и леммы 4.

5. Доказательства теорем. Отметим сначала, что из обобщенного закона арксинуса и определения (6) событий $A_{i}(n), i=1,2,3,4$, следует, что

$$
\begin{aligned}
\lim _{n \rightarrow \infty} \mathbf{P}\left(A_{1}(n)\right) & =\mathbf{P}\left(\tau \leqslant t_{1}\right), \quad \lim _{n \rightarrow \infty} \mathbf{P}\left(A_{2}(n)\right)=\mathbf{P}\left(\tau \in\left[t_{1}, t_{2}\right]\right), \\
\lim _{n \rightarrow \infty} \mathbf{P}\left(A_{3}(n)\right) & =\lim _{n \rightarrow \infty} \mathbf{P}\left(\tau\left(n t_{2}\right) \leqslant n t_{1}\right)-\lim _{n \rightarrow \infty} \mathbf{P}\left(\tau(n) \leqslant n t_{1}\right) \\
& =\mathbf{P}\left(\tau \leqslant \frac{t_{1}}{t_{2}}\right)-\mathbf{P}\left(\tau \leqslant t_{1}\right), \\
\lim _{n \rightarrow \infty} \mathbf{P}\left(A_{4}(n)\right) & =\lim _{n \rightarrow \infty} \mathbf{P}\left(\tau\left(n t_{2}\right)>n t_{1}\right)-\lim _{n \rightarrow \infty} \mathbf{P}\left(\tau(n) \in\left[n t_{1}, n t_{2}\right]\right) \\
& =\mathbf{P}\left(\tau>\frac{t_{1}}{t_{2}}\right)-\mathbf{P}\left(\tau \in\left[t_{1}, t_{2}\right]\right) .
\end{aligned}
$$

Таким образом, $\lim _{n \rightarrow \infty} \mathbf{P}\left(A_{i}(n)\right)>0, i=1,2,3,4$. Откуда вытекает, что для любого допустимого события $D(n)$

$$
\lim _{n \rightarrow \infty} \mathbf{P}\left(D(n) \mid A_{i}(n)\right)=0 \Longleftrightarrow \lim _{n \rightarrow \infty} \mathbf{P}\left(D(n) ; A_{i}(n)\right)=0 .
$$

В этом пункте мы будем часто использовать этот факт без дополнительньх пояснений.

Доказательст во теоремы 2. Для $m, n \in \mathbf{N}, m<n$, положим

$$
\alpha_{n}(m):=\frac{1-f_{0, m}(0)}{1-f_{0, n}(0)} .
$$

Очевидно, что $\alpha_{n}(m) \geqslant 1$. Введем функцию

$$
h^{(n)}(s)=h_{t_{1}, t_{2}}^{(n)}(s):=\frac{f_{0, n t_{1}}\left(a+s y_{2}\right)-f_{0, n t_{1}}(0)}{1-f_{0, n}(0)}
$$

(см. (10) и (11)). Несложно заметить, что $h^{(n)}(s)$ является производящей функцией (неотрицательной) меры на $\mathrm{N}_{0}, \mathrm{c}$ весом

$$
h^{(n)}(1)=\frac{f_{0, n}(0)-f_{0, n t_{1}}(0)}{1-f_{0, n}(0)}=\alpha_{n}\left(n t_{1}\right)-1
$$


Пусть

$$
\begin{aligned}
T^{(n)}(\lambda)=T_{t_{1}, t_{2}}^{(n)}(\lambda) & :=\frac{f_{0, n t_{1}}\left(a+e^{-\lambda \Theta_{n}\left(t_{1}, t_{2}\right)} y_{1}\right)-f_{0, n t_{1}}(0)}{1-f_{0, n t_{1}}(0)} \\
& =: \int_{0}^{\infty} e^{-\lambda x} \mathscr{T}^{(n)}(d x)
\end{aligned}
$$

И

$$
\begin{aligned}
H^{(n)}(\lambda)=H_{t_{1}, t_{2}}^{(n)}(\lambda) & :=h^{(n)}\left(e^{-\lambda \Theta_{n}\left(t_{1}, t_{2}\right)}\right) \\
& =: \int_{0}^{\infty} e^{-\lambda x} \mathscr{H}_{t_{1}, t_{2}}^{(n)}(d x)=\int_{0}^{\infty} e^{-\lambda x} \mathscr{H}^{(n)}(d x) .
\end{aligned}
$$

Легко видеть, что $\mathscr{T}^{(n)}$ и $\mathscr{H}^{(n)}$ являются положительными мерами такими, что

$$
H^{(n)}(0)=\int_{0}^{\infty} \mathscr{H}^{(n)}(d x)=\alpha_{n}\left(n t_{1}\right)-1, \quad T^{(n)}(0)=\int_{0}^{\infty} \mathscr{T}^{(n)}(d x)=1 .
$$

Пусть

$$
d\left(\mathscr{M}^{(n)}, \mathscr{T}^{(n)}\right):=\frac{1}{2} \int_{0}^{\infty}\left|\mathscr{M}^{(n)}(d x)-\mathscr{T}^{(n)}(d x)\right|
$$

- расстояние по вариации между мерами $\mathscr{M}^{(n)}$ и $\mathscr{T}^{(n)}$. Учитывая (12), несложно понять, что

$$
M_{t_{1}, t_{2}}^{(n)}(\lambda)=m^{(n)}\left(e^{-\lambda \Theta_{n}\left(t_{1}, t_{2}\right)}\right)=\alpha_{n}\left(n t_{1}\right) T^{(n)}(\lambda)-H^{(n)}(\lambda) .
$$

Следовательно, для доказательства теоремы достаточно показать, что для любого $\varepsilon>0$

$$
\lim _{n \rightarrow \infty} \mathbf{P}\left(d\left(\mathscr{M}^{(n)}, \mathscr{T}^{(n)}\right)>\varepsilon \mid A_{1}(n)\right)=0
$$

И

$$
T^{(n)}(\lambda) \stackrel{w_{A_{1}}(\mathbf{P}, \widehat{\mathbf{P}})}{\longmapsto} L(\lambda)
$$

Из (38) и (39) получаем

$$
\begin{aligned}
2 d\left(\mathscr{M}^{(n)}, \mathscr{T}^{(n)}\right) & =\int_{0}^{\infty}\left|\left(\alpha_{n}\left(n t_{1}\right)-1\right) \mathscr{T}^{(n)}(d x)-\mathscr{H}^{(n)}(d x)\right| \\
& \leqslant\left(\alpha_{n}\left(n t_{1}\right)-1\right) \int_{0}^{\infty} \mathscr{T}^{(n)}(d x)+\int_{0}^{\infty} \mathscr{H}^{(n)}(d x) \\
& \leqslant 2\left(\alpha_{n}\left(n t_{1}\right)-1\right) .
\end{aligned}
$$

Согласно следствию 3 работы [10], для любого $\varepsilon>0$

$$
\lim _{n \rightarrow \infty} \mathbf{P}\left(\left|\alpha_{n}\left(n t_{1}\right)-1\right|>\varepsilon \mid A_{1}(n)\right)=0
$$

Объединяя это соотношение с (42), приходим к (40). 
Согласно определению сходимости $\stackrel{w_{A_{1}}(\mathbf{P}, \widehat{\mathbf{P}})}{\longrightarrow}$, для доказательства (41) нужно показать, что для любых неслучайных непрерывных функций $J, g: \mathbf{R} \rightarrow \mathbf{R}$ с компактными носителями

$$
\lim _{n \rightarrow \infty} \mathbf{E}\left[g\left(\mathscr{T}^{(n)}[J]\right) \mid A_{1}(n)\right]=\widehat{\mathbf{E}}[g(\mathscr{L}[J])]
$$

Несложно проверить, используя (30) и (37), что для $l>0$

$$
\mathbf{E}\left[g\left(\mathscr{T}^{(n)}[J]\right) \mid \tau(n)=l<n t_{1}\right]=\mathbf{E}\left[g\left(\mathscr{T}_{n t_{1}-l}^{\left(l, n t_{2}-l\right)}[J]\right) \mid \mathscr{A}_{l, n-l}\right],
$$

где $\mathscr{T}_{m}^{(l, k)}$ - мера с преобразованием Лапласа $T_{m}^{(l, k)}(\lambda)$, определенным равенством (30), и $\mathscr{A}_{l, n-l}=\left\{\Gamma^{-}>l, \gamma^{+}>n-l\right\}$ (см. (3)). Согласно лемме $5, \mathscr{T}_{m}^{\left(l, n t_{2}-l\right)}[J] \rightarrow \mathscr{L}[J] \widehat{\mathbf{P}}$-п.н. при $\min (l, m) \rightarrow \infty, m<n t_{2}-l$. Отсюда и из леммы 3 работы [10] вытекает, что для любых неслучайных функций $J, g: \mathbf{R} \rightarrow \mathbf{R}$ с компактными носителями

$$
\begin{aligned}
& \lim _{\min \left(l, n t_{1}-l\right) \rightarrow \infty} \mathbf{E}\left[g\left(\mathscr{T}^{(n)}[J]\right) \mid \tau(n)=l<n t_{1}\right] \\
& \quad=\lim _{\min \left(l, n t_{1}-l\right) \rightarrow \infty} \mathbf{E}\left[g\left(\mathscr{T}_{n t_{1}-l}^{\left(l, n t_{2}-l\right)}[J]\right) \mid \mathscr{A}_{l, n-l}\right]=\widehat{\mathbf{E}}[g(\mathscr{L}[J])] .
\end{aligned}
$$

Для завершения доказательства (43) осталось использовать при $\varepsilon \in$ $\left(0, \frac{1}{2}\right)$ формулу полной вероятности

$$
\begin{aligned}
& \mathbf{E}\left[g\left(\mathscr{T}^{(n)}[J]\right) ; A_{1}(n)\right]=\sum_{0 \leqslant l / t_{1} \leqslant n \varepsilon} \mathbf{E}\left[g\left(\mathscr{T}_{n t_{1}-l}^{\left(l, n t_{2}-l\right)}[J]\right) \mid \mathscr{A}_{l, n-l}\right] \mathbf{P}(\tau(n)=l) \\
& \quad+\sum_{n \varepsilon<l / t_{1}<n(1-\varepsilon)} \mathbf{E}\left[g\left(\mathscr{T}_{n t_{1}-l}^{\left(l, n t_{2}-l\right)}[J]\right) \mid \mathscr{A}_{l, n-l}\right] \mathbf{P}(\tau(n)=l) \\
& \quad+\sum_{n(1-\varepsilon) \leqslant l / t_{1} \leqslant n} \mathbf{E}\left[g\left(\mathscr{T}_{n t_{1}-l}^{\left(l, n t_{2}-l\right)}[J]\right) \mid \mathscr{A}_{l, n-l}\right] \mathbf{P}(\tau(n)=l)
\end{aligned}
$$

устремить $n$ к бесконечности, заметить, что первая и третья суммы пренебрежимо малы в силу ограниченности функции $g$ и обобщенного закона арксинуса (см. (7)), согласно которому

$$
\lim _{\varepsilon \downarrow 0} \lim _{n \rightarrow \infty} \mathbf{P}\left(\tau(n) \in\left[0, n t_{1} \varepsilon\right] \bigcup\left[n t_{1}(1-\varepsilon), n t_{1}\right]\right)=0
$$

а затем оценить вторую сумму, опираясь на (44).

Теорема 2 доказана.

Дока зательст в о те о ре мы 3. Введем функции

$$
\begin{gathered}
T^{(n) *}(\lambda)=T_{t_{1}, t_{2}}^{(n) *}(\lambda):=\frac{f_{0, n t_{1}}^{\prime}\left(1-\left(1-e^{-\lambda \Theta_{n}\left(t_{1}, t_{2}\right)}\right)\left(1-f_{n t_{1}, n t_{2}}(0)\right)\right)}{e^{S_{n t_{1}}}}, \\
W^{(n) *}(\lambda)=W_{t_{1}, t_{2}}^{(n) *}(\lambda):=T^{(n) *}(\lambda) e^{-\lambda \Theta_{n}\left(t_{1}, t_{2}\right)}=: \int_{0}^{\infty} e^{-\lambda x} \mathscr{W}^{(n) *}(d x) .
\end{gathered}
$$


Легко видеть, что функция $T^{(n) *}(\lambda)$ и определенная в (37) функция $T^{(n)}(\lambda)$ являются аналитическими в области $\operatorname{Re} \lambda>0$ и

$$
T^{(n) *}(\lambda)=-\frac{d}{d \lambda} T^{(n)}(\lambda)
$$

По лемме 6 для доказательства теоремы достаточно установить, что (см. теорему 1)

$$
W_{t_{1}, t_{2}}^{(n) *}(\lambda) \stackrel{w_{A_{3}}(\mathbf{P}, \widehat{\mathbf{P}})}{\longrightarrow} W(\lambda), \quad \lambda>0 .
$$

Таким образом, нужно показать, что для любых неслучайных непрерывных функций $J, g: \mathbf{R} \rightarrow \mathbf{R}$ с компактными носителями

$$
\lim _{n \rightarrow \infty} \mathbf{E}\left[g\left(\mathscr{W}^{(n) *}[J]\right) \mid A_{3}(n)\right]=\widehat{\mathbf{E}}[g(\mathscr{W}[J])]
$$

Очевидно, что

$$
\begin{aligned}
\mathbf{E}\left[g\left(\mathscr{W}^{(n) *}[J]\right) ; A_{3}(n)\right]= & \mathbf{E}\left[g\left(\mathscr{W}^{(n) *}[J]\right) ; \tau\left(n t_{2}\right) \leqslant \tau\left(n t_{1}\right)\right] \\
& -\mathbf{E}\left[g\left(\mathscr{W}^{(n) *}[J]\right) ; \tau(n) \leqslant n t_{1}\right] .
\end{aligned}
$$

Докажем, что

$$
\lim _{n \rightarrow \infty} \mathbf{E}\left[g\left(\mathscr{W}^{(n) *}[J]\right) ; \tau(n) \leqslant n t_{1}\right]=\widehat{\mathbf{E}}[g(\mathscr{W}[J])] \mathbf{P}\left(\tau \leqslant t_{1}\right)
$$

Действительно, несложно проверить, что

$$
\mathbf{E}\left[g\left(\mathscr{W}^{(n) *}[J]\right) ; \tau(n)=l<n t_{1}\right]=\mathbf{E}\left[g\left(\mathscr{W}^{\left(l, n t_{1}-l, n t_{2}-\cdot, \cdot *\right.}[J]\right) \mid \mathscr{A}_{l, n-l}\right]
$$

где

$$
T_{n t_{1}-l}^{\left(l, n t_{2}-l\right) *}(\lambda) e^{-\lambda \Theta\left(l, n t_{1}-l, n t_{2}-l\right)}=: \int_{0}^{\infty} e^{-\lambda x} \mathscr{W}^{\left(l, n t_{1}-l, n t_{2}-l\right) *}(d x)
$$

Согласно лемме $6, \mathscr{W}^{(l, m, k) *}[J] \rightarrow \mathscr{W}[J] \widehat{\mathbf{P}}$-п.н. при $\min (l, m) \rightarrow \infty, m<k$. Отсюда и из леммы 3 в [10] следует, что для любых неслучайных непрерывных функций $J, g: \mathbf{R} \rightarrow \mathbf{R}$ с компактными носителями

$$
\begin{aligned}
& \lim _{\min \left(l, n t_{1}-l\right) \rightarrow \infty} \mathbf{E}\left[g\left(\mathscr{W}^{(n) *}[J]\right) \mid \tau(n)=l<n t_{1}\right] \\
& \quad=\lim _{\min \left(l, n t_{1}-l\right) \rightarrow \infty} \mathbf{E}\left[g\left(\mathscr{W}^{\left(l, m, n t_{2}-l\right) *}[J]\right) \mid \mathscr{A}_{l, n-l}\right]=\widehat{\mathbf{E}}[g(\mathscr{W}[J])]
\end{aligned}
$$

При помощи (49) несложно прийти к (48), применяя рассуждения, использованные при выводе (43) из соотношения (44). Используя аналогичные соображения, можно показать, что

$$
\lim _{n \rightarrow \infty} \mathbf{E}\left[g\left(\mathscr{W}^{(n) *}[J]\right) ; \tau\left(n t_{2}\right) \leqslant n t_{1}\right]=\widehat{\mathbf{E}}[g(\mathscr{W}[J])] \mathbf{P}\left(\tau \leqslant \frac{t_{1}}{t_{2}}\right)
$$


Теперь соотношения (47)-(50), свойство (36) и разложение $\mathbf{E}\left[g\left(\mathscr{W}^{(n) *}[J]\right)\right.$; $\left.A_{3}(n)\right]$ на три слагаемых, аналогичное (45), влекут (46).

Теорема 3 доказана.

Доказ ательст во те о ремы 4. В силу леммы 3 достаточно убедиться в том, что при $n \rightarrow \infty$

$$
m^{(n) *}(s) \stackrel{w_{A_{i}}(\mathbf{P}, \widehat{\mathbf{P}})}{\longmapsto} s, \quad i=2,4 .
$$

Пусть $\delta_{1}(\{1\})=1, \delta_{1}(\{k\})=0$ при $k \neq 1, k \in \mathbf{N}_{0}$, - вероятностная мера на $\mathbf{N}_{0}$ с единичным атомом в точке 1 . Оценим расстояние по вариации $d\left(\delta_{1}, \mu^{(n) *}\right)$ между мерой $\delta_{1}$ и мерой $\mu^{(n) *}$, введенной в (17). Заметим, что для доказательства (51) достаточно проверить, что для любого $\varepsilon>0$

$$
\lim _{n \rightarrow \infty} \mathbf{P}\left(d\left(\delta_{1}, \mu^{(n) *}\right)>\varepsilon \mid A_{i}(n)\right)=0, \quad i=2,4,
$$

или, ввиду представления $\left\{\tau\left(n t_{2}\right)>n t_{1}\right\}=A_{2}(n) \cup A_{4}(n)$, что

$$
\lim _{n \rightarrow \infty} \mathbf{P}\left(d\left(\delta_{1}, \mu^{(n) *}\right)>\varepsilon \mid \tau\left(n t_{2}\right)>n t_{1}\right)=0 .
$$

Ясно, что

$$
d\left(\delta_{1}, \mu^{(n) *}\right)=\frac{1}{2} \sum_{k=1}^{\infty}\left|\mu^{(n) *}(k)-\delta_{1}(k)\right|=1-\mu^{(n) *}(1) .
$$

Из соотношения (17), определяющего меру $m^{(n) *}(s)$, следует, что

$$
\mu^{(n) *}(1)=\frac{f_{0, n t_{1}}^{\prime}\left(f_{n t_{1}, n t_{2}}(0)\right)}{e^{S_{n t_{1}}}}
$$

Ввиду неравенств $e^{S_{n t_{1}}}-f_{0, n t_{1}}^{\prime}(s) \leqslant f_{0, n t_{1}}^{\prime \prime}(1)(1-s)$ и $1-f_{0, n t_{2}}(0) \leqslant e^{S_{\tau\left(n t_{2}\right)}}$ имеем

$$
\begin{aligned}
1-\mu^{(n) *}(1) & \leqslant \frac{f_{0, n t_{1}}^{\prime \prime}(1)\left(1-f_{n t_{1}, n t_{2}}(0)\right)}{e^{S_{n t_{1}}}} \\
& =2 b_{n t_{1}} e^{S_{n t_{1}}}\left(1-f_{n t_{1}, n t_{2}}(0)\right) \\
& =2 b_{n t_{1}}\left(1-f_{0, n t_{2}}(0)\right) \sigma_{n t_{2}}\left(n t_{1}\right) \leqslant \sigma_{n t_{2}}\left(n t_{1}\right) e^{\left.S_{\tau\left(n t_{2}\right)}\right)} b_{n t_{1}},
\end{aligned}
$$

где случайная величина $b_{r}$ та же, что и в (23). По лемме 1

$$
\left\{\sigma_{n t_{2}}\left(n t_{1}\right) \mid \tau\left(n t_{2}\right)>n t_{1}\right\} \stackrel{d}{\longmapsto} 1 .
$$

С другой стороны, соотношение (27) дает

$$
\lim _{n \rightarrow \infty} \mathbf{P}\left(e^{S_{\tau\left(n t_{2}\right)}} b_{n t_{1}} \mid \tau\left(n t_{2}\right)>n t_{1}\right)=0 .
$$

Объединяя (53)-(56), получаем (52), что, как мы видели, влечет (51).

Теорема 4 доказана. 


\section{СПИСОК ЛИТЕРАТУРЫ}

1. Athreya K.B., Karlin S. On branching processes with random environments. I: Extinction probability. - Ann. Math. Statist., 1971, v. 42, № 5, p. 1499-1520.

2. Athreya $K$. B., Karlin $S$. On branching processes with random environments. II: Limit theorems. - Ann. Math. Statist., 1971, v. 42, № 6, p. 1843-1858.

3. Athreya K.B., Ney P.E. Branching Processes. New York-Heidelberg: SpringerVerlag, 1972, $287 \mathrm{p}$.

4. Afanasyev V.I., Geiger J., Kersting G., Vatutin V. A. Criticality for branching processes in random environment. - Ann. Probab., 2005, v. 33, № 2, p. 645-673.

5. Borovkov K.A., Vatutin V.A. Reduced critical branching processes in random environment. - Stochastic Process. Appl., 1997, v. 71, № 2, p. 225-240.

6. Ватутин $B$. A. Редуцированные ветвящиеся процессы в случайной среде. - Теория вероятн. и ее примен., 2002, т. 47, в. 1, с. 21-38.

7. Vatutin V.A., Dyakonova E.E. Reduced branching processes in random environment. - Mathematics and Computer Science. II: Algorithms, Trees, Combinatorics and Probabilities. Ed. by B. Chauvin et al. Basel: Birkhäuser, 2002, p. 455-467.

8. Vatutin V. A., Dyakonova E. E. Yaglom type limit theorem for branching processes in random environment. - Mathematics and Computer Science. III: Algorithms, Trees, Combinatorics, and Probabilities. Ed. by M. Drmota et al. Basel: Birkhäuser, 2004, p. 375-385.

9. Ватутин B. A., Дьяконова E. E. Ветвящиеся процессы Гальтона-Ватсона в случайной среде. I: предельные теоремы. - Теория вероятн. и ее примен., 2003, т. 48, в. 2 , c. $274-300$.

10. Ватутин B. A., Дьяконова E. E. Ветвящиеся процессы Гальтона-Ватсона в случайной среде. II: конечномерные распределения. - Теория вероятн. и ее применен., 2004, т. 49 , в. 2 , с. 231-268.

11. Ватутин B.A., Дьяконова E.E. Ветвяшиеся процессы в случайной среде и бутылочные горлышки в эволюции популяций. - Теория вероятн. и ее примен., 2006 , т. 51 , в. 1, c. $22-46$.

12. Ватутин В.А., Дьяконова E.E. Предельные теоремы для редуцированных ветвяшихся процессов в случайной среде. - Теория вероятн. и ее примен., 2007, т. 52 , в. 2 , c. $271-300$.

13. Doney R. A. Spitzer's condition and the ladder variables in random walks. - Probab. Theory Related Fields, 1995, v. 101, № 4, p. 577-580.

14. Зубков А.М. Предельные распределения расстояния до ближайшего общего предка. - Теория вероятн. и ее примен., 1975, т. 20, в. 3, с. 614-623.

15. Спичер Ф. Принципы случайного блуждания. М.: Мир, 1969, 472 с.

16. Fleischmann K., Vatutin V.A. Reduced subcritical Galton-Watson processes in a random environment. - Adv. Appl. Probab., 1999, v. 31, № 1, p. 88-111.

17. Fleischmann $K$., Prehn U. Ein Grenzwertsatz für subkritische Verzweigungsprozesse mit endlich vielen Typen von Teilchen. - Math. Nachr., 1974, v. 64, p. 357-362.

18. Fleischmann K., Siegmund-Schultze R. The structure of reduced critical GaltonWatson processes. - Math. Nachr., 1977, v. 79, p. 233-241.

Поступила в редакцию 23.IV.2007 